\title{
Hipoplasia Femoral y Facies Especial. Un Síndrome de Expresividad Variable
}

\author{
Dra. Eliana Selman C. ${ }^{1}$; Dr. Andrés Cantín D.2; Dr. Aldo Bancalari M. ${ }^{3}$ \\ Femoral Hypoplasia - Unusual Facies. \\ A Syndrome of Variable Expressivity
}

\begin{abstract}
Two patients bearing the femoral hypoplasia - unusual facies syndrome are reported. Both were born from young mothers and had no rejevant familiar background. Their phenotypical traits are described and compared with those already published. The variable expressivity of this syndrome has been enphasized. Besides, some aspects of hereditary mechanism of this syndrome are also discussed.
\end{abstract}

El síndrome de hipoplasia femoral y facies especial fue descrito por primera vez por Daentl ${ }^{1}$ en 1975, quien tiene el mérito de haber asociado las alteraciones faciales y de las extremidades estructurándolas en una sola entidad, cuyos hechos aislados habian sido publicados por Franz y O'Rahilly en 1961 y Kucera en 1965. Se caracte. riza por hipoplasia proximal del fémur asociada a

1 Unidad de Genética, Servicio de Pediatria, Hospital Guillermo Grant Benavente, de Concepción.

2 Servicio de Radiología. Hospital Guillermo Grant Benavente, de Concepción.

3 Unidad de Neonatología. Servicio de Pediatria. Hospital Guillermo Grant Benavente, de Concepción. algunas características faciales como fisuras palpebrales "mongoloides", nariz pequeña con alas hipoplásticas, Iabio superior fino, filtrum largo y en algunos, fisura palatina. Se pueden encontrar otras alteraciones esqueléticas especialmente a nivel de pelvis, columna lumbar y extremidades.

En la literatura existen aproximadamente 40 pacientes publicados ${ }^{2-3-4-5}$, observándose en la mayoría una variable expresividad en cuanto a sus manifestaciones clínicas y sin una etiología clara, ya que en su mayoría representan casos esporádicos.

El propósito de esta comunjcación es mostrar dos pacientes con hipoplasia femoral unilateral, con las características faciales descritas en el 
sindrome y compararlas con los anteriomente publicados. También discutiremos algunos aspectos en cuanto a su posible mecanismo de herencia.

\section{Caso 1. (Fig. 1).}

RN de pretérmino de sexo femenino, primer hijo de madre de 18 años, sana y sin antecedentes familiares de importancia. El padre, 30 años de edad. Se desconocen sus antecedentes familiares. El embarazo transcurrió sin patología. Parto con cesárea debido a presentación podálica en el octavo mes de gestación. Nació con puntaje Apgar 6 al 1' y 8 a los 3', lo que se cataloga como assfixia leve. Presentó un peso al nacer de 1920 gramos, una talla de $44 \mathrm{~cm}$. y una circunferencia de cráneo de $32 \mathrm{~cm}$. El examen neurológico era normal. Presentó ictericia fisiológica desde el $4^{\circ}$ día de vida que se trató con luminoterapia.

Examen Físico a los 6 meses: Cráneo de conformación normal, orejas bien implantadas sin malformaciones; hipertelorismo, ojos con abertura palpebral tmongoloide, nariz corta y angosta, labio superiur fino $y$ largo, paladar ojival y una moderada micrognasia. Extremidades supe. ríres: En la mano derecha se observa sindactilia cutánea de los dedos medio y anular y ausencia del meñique. El estudio radiológico a ese nivel comprueba agenesia del $5^{\circ}$ metacarpiano y del

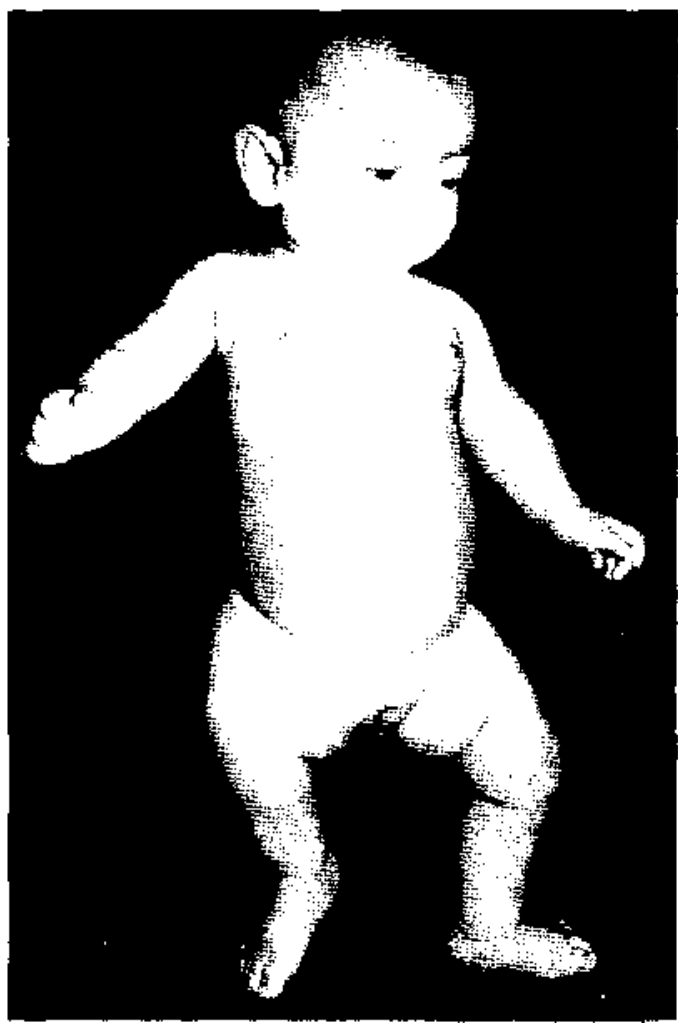

Figura I. dedo meñique. Fig. 2. Extremidades inferiores: notoria asimetría debida a marcado acortamiento del muslo derecho. La radiología demuestra acentuada hipoplasia del fémur proximal derecho con aparente indemnidad del techo acetabular. No se ve aún el núcleo de osificación de la cabeza femoral. Extremidad inferior izquierda normal.

El control radiológico a los 4 años revela un techo acetabular derecho discretarnente diplásico ahora se ve la cabeza femoral en la cavidad articular, separada de la metadiáfisis femoral que es hipoplásica. (Tipo B de Levinson ${ }^{7}$ ) (Fig. 3). Se aprecia además discreta hipoplasia del peroné ipsilateral. La paciente en la actualidad tiene 4 afios 6 meses, desarrollo pontoestatural y sicomotor normales. Camina desde $\operatorname{los} 2$ anos ayudada por un zapato especial.

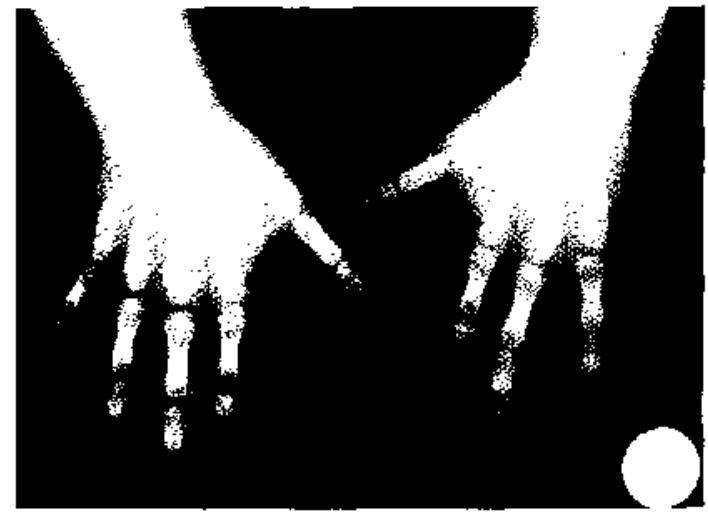

Figura 2. Caso 1. Se observa agenesia del $5^{\circ}$ metacarpiano y del meñitjue.

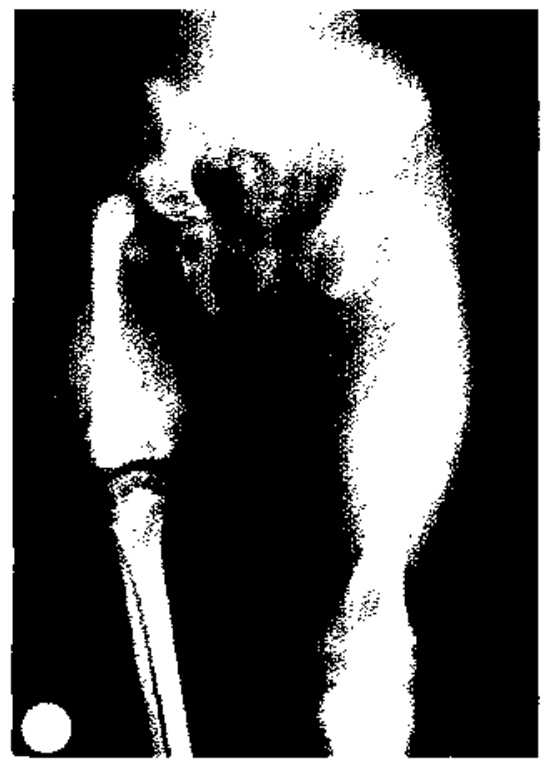

Finura 3. Caso 1. Se observa hipoplasia femotal derecha con techo acetabular ipsilateral moderadamente displisicn. 


\section{Caso 2. (Fig. 4)}

Recién nacido, de término de sexo masculino, primer hijo de madre soltera de 21 años de edad, sana y sin antecedentes familiares de importancia. Padre de aproximadamente 40 años, sano, bebedor moderado, tiene 3 hijos sanos de su matrimonio legal, desconociéndose otros antecedentes farniliares. Embarazo sin patología y sin antecedentes de ingesta de drogas. El parto fue por cesárea debido a presentación podálica a las 40 semanas de gestación. Puntaje Apgar 5 al $1^{\prime} \mathrm{y}$ 8 a los 3', peso de 2.920 gramos, talla $52 \mathrm{~cm}$. $y$ cc de $35,5 \mathrm{~cm}$. Examen neurológico normal. Examen Físico: Cráneo de conformación normal, aberturas palpebrales mongoloides, nariz pequeña y alas hipoplásicas, Jabio superior fino, filtrum largo y micrognasia moderada. Las extremidades inferiores muestran marcada asimetria, notorio acortamiento del muslo derecho y en la radiología hipoplásica proximal del fémur derecho $y$ configuración normaldel techo acetabular (Tipo $A$ de Lenvinson ${ }^{7}$ ). Peroné ipsilateral modera. damente hipoplásico. Resto del esqueleto óseo normal. (Fig. 5).

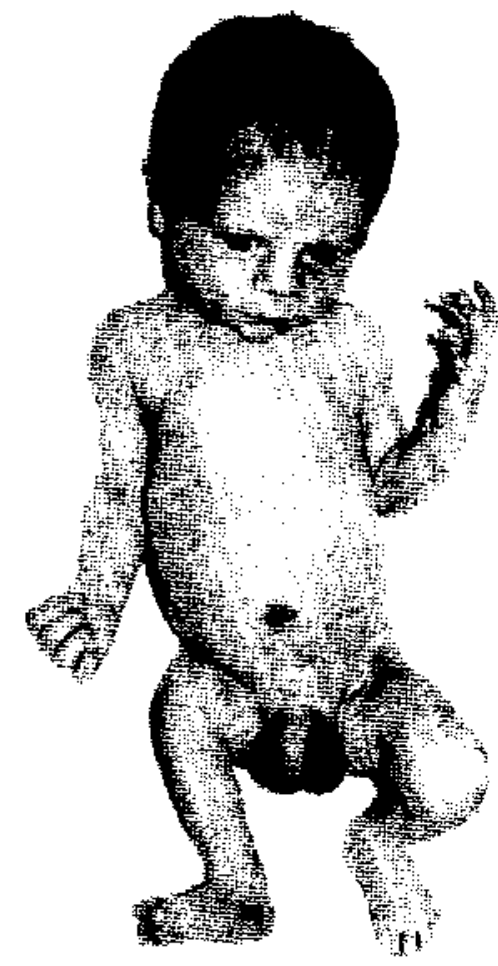

Figura 4, Caso 2.

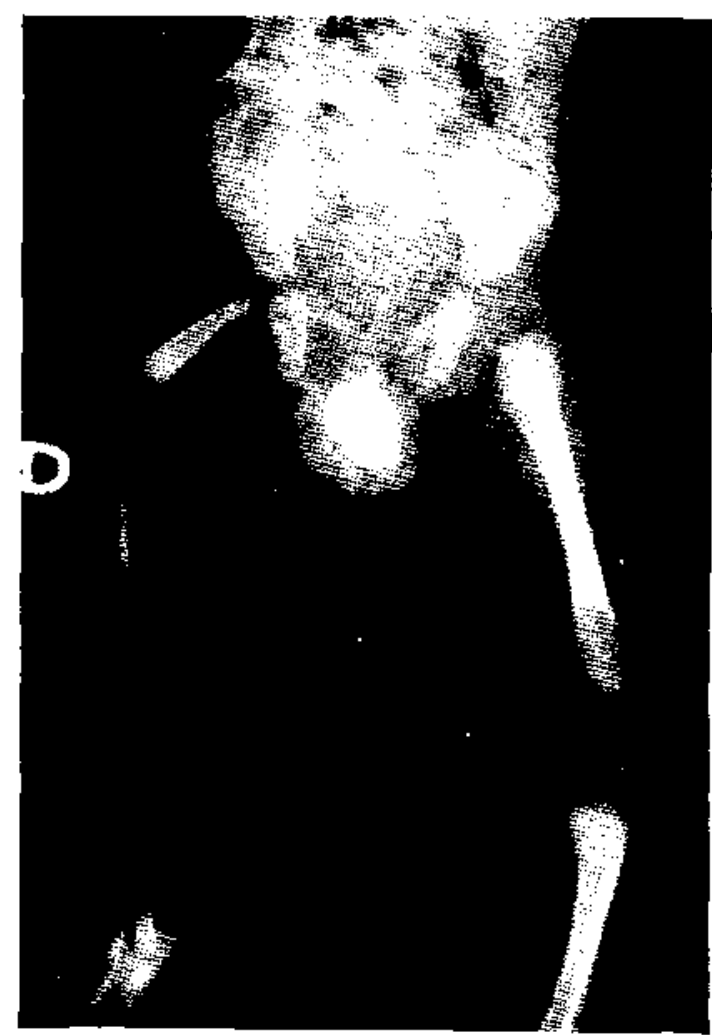

Figura 5. Caso 2. Se observa hipoplasiz proximal del fémur derecho y configuración normal del techo acetabular.

\section{COMENTARJO}

La lipoplasia femoral o deficiencia focal proximal del fémur, como es conocida por los ortopedistas, es una rara malformación, pero ha sido estudiada en detalle 6 . Puede ser unilateral o bilateral y, dependiendo de la distribución anatómica de la anormalidad, se puede clasificar según Levinson en los tipos: $\mathrm{A}-\mathrm{B}-\mathrm{C}$ y $\mathrm{D}^{7}$.

El primero en plantear una entidad sindrómica para esta alteración fue Daentl et al. en 1975, publicando una serie de 4 pacientes que presentaban la hipoplasia del fémur asociada a una facies especial cuyas características fueron especificadas anteriormente. Posteriormente diversos autores han comunicado varios casos de este síndrome, apreciándose una expresividad muy variable de sus características fenotípicas, tanto faciales como esqueléticas. Todos los casos fueron bilaterales, excepto el de Graviss y $\mathrm{Col}$ en $1980^{\circ}$ que es unilateral, pero fue presentado por dicho autor como hipoplasia femoral asociada a Secuencia de Pierre-Robin; sin embargo no estamos de acuerdo con esta asociación y pensamos que corresponde al mismo síndrome presentado par noso- 
tros, mostrando una forna intermedia de expresión de la anomalia.

Johnson en $1983^{5}$ plantea un criterio mínimo para el diagnóstico de esta entidad consistente en la presencia de hipoplasia del fémur y de por lo menos 2 de las características faciales. El concluye que el sindrome completo puede representar la más severa expresión de una malformación compleja siendo la hipoplasia femoral aislada una manifestación mínima. Nuestro primer caso podría corresponder a una forma intermedia, ya que presenta hipoplasia femoral derecha tipo B, algunas características faciales y alteraciones esqueléticas como agenesia del $5^{\circ}$ metacarpiano, del dedo meñique de la mano e hipoplasia del peroné ipsilaterales. El segundo caso es fenotipicamente menos afectado, ya que la hipoplasia femoral es del tipo A y sus alteraciones facjales son moderadas, por lo que correspondería a una forma mínima de expresión del síndrome.

Todas las publicaciones menos una, coinciden en que la afección es esporádiça. La única excep. ción fue comunicada por Lampert en $1980^{\circ}$, quien observó el caso de un padre y su hija, lo que sugiere una herencia autosómica dominante. Sin embargo, es rato que siendo éste el mecanismo de herencia, no existan otros ejemplos similares. Podria pensarse que todos sean producto de una mutación. Estaría en contra el hecho de encontrar, en una serie publicada por Lord y Beighton $^{10}$, un paciente con el síndrome que habia procreado 5 hijos normales.

Tampoco hay antecedentes en la literatura ni en nuestros casos de ingestión de teratógenos durante el embarazo, por lo que se descarta que este factor, tenga un rol etiopatogénico en el sindrome.

El estudio cromosómico practicado en varios pacientes comunicados anteriormente $\mathrm{e}^{3-5-8}$, ha dado resultados normales, haciendo poco probable que una aberración cromosómica sea la causa de la entidad.

Por otra parte, la baja frecuencia de parientes afectados con una ocasional transmisión de padre a hijo, sería más sugerente de un tipo de herencia multifactorial o poligénica, como plantean Lord $y$ Beighton ${ }^{10}$. Por último, el hecho de que 15 pacientes descritos por los diversos autores, tienen el antecedente de diabetes en la madre 6 y que no se observó en riuestros 2 casos, sugiere que la diabetes materna podría ser uno de los factores ambientales desencadenantes en un modelo de herencia multifactorial.

\section{RESUMEN}

Se presentan 2 pacientes portadores del Sindrome de Hipoplasia Femoral/Facies especial. Ambos eran hijos de madres jóvenes y sin antecedentes familiares de importancia. Se describen sus características fenotipicas y se comparan con los anteriomente publicados, enfatizándose la variable expresividad del sindrome. Se discuten algunos aspectos referentes a su posible mecanismo de herencia.

\section{REFERENCIAS}

${ }^{1}$ Daenti, D.L.; Smith D.W., Scott C.I., Hall B.D., Gooding $C A_{\text {A.: }}$ Fcmoral hypoplasia - unusual facies syndrome. J. Pediatrics 86: 107, 1975.

${ }^{2}$ Hurst D., Johnson D.F.: Brief clinical report: Femoral hypoplasia unusual facies syndroms. Am. J. Med. Genet. 5: 255, 1980.s,

3 Holmes L.B.: Femoral hypoplasia - unusual facies syndrome. J. Pediatrics 87: 668, 1975.

${ }^{4}$ Gleiser S. Weaver D.D., Escobar B., Nichols, G. Escobedo M.: Femoral hypoplasia - unusual facies syndrome, from another viewpoint. Eur. J. Pediatr. 128: 1,1978

5 Johnson, J.P., Carey J.C., Manford G. Petersen, J. and Beattie J.F.: Femotal hypoplasia - unusual facies syndrome in infants of diabetic mothers. J. Pediatr. 102: 866, 1983.

${ }^{6}$ Aitken G.T.: Proximal focal deficiency: A congenital anomaly. Symposium, National Academy of Sciences, Washington, D.C. Junio $13,1968$.

${ }^{7}$ Levinson E.D., Ozanoff M.B., Royen P.M.: Proximal femoral focal deficiency (PFFD) Radiology 125: 197, 1977.

Graviss R.E., Monteleone P.A., Wampler L.R., Silberstein M.J., Brodeur A.E.: Proximal femoral focal deficiency. Associated with the Robin anomalad. J. Med. Genet. 17; 390, 1980.

${ }^{9}$ Lampert, R.P.: Dominant, inheritance of femoral liypoplasia-unusual facies syndrome. Clin. Genet. 17, 255-258.

10 Lord J. Beighton P.: The femoral hypoplasia -unusual facies syndrome: a genctic entity? Clin. Genet. 20: 267, 1981. 